\title{
Die Verzerrungen auf alten Seekarten und ihre Deutung
}

\author{
Von Heinz Balmer
}

\section{Beschreibung der Verzerrungen}

In den fünf Bänden der Portugaliae Monumenta Cartographica (Lissabon 1960) liegt die größte Sammlung portugiesischer Seekarten aus dem 16. und 17. Jahrhundert vor. Sie wurde mir von Prof. Dr. Fritz Gygax in Bern zur Verfügung gestellt und diente zur Grundlage der vorliegenden Arbeit. Es wurden einzelne Karten ausgewählt und mit dem Verfahren der Verzerrungsgitter (wie es Eduard Imhof auf Binnenlandkarten anwandte) auf ihre Genauigkeit geprüft.

Dabei wird ein halbdurchsichtiges Papier über die alte Karte gespannt und mit Hilfe eines neuen Atlasses das Netz der Längen- und Breitenkreise auf die alte Karte übertragen. Die Linien werden durch die richtigen Orte gezogen und erleiden dabei mehr oder weniger starke Krümmungen. Sie biegen sich auf und nieder, wo Orte gleicher Breite nicht waagrecht nebeneinanderstehen, oder hin und her, wo Orte gleicher Länge nicht senkrecht untereinander gesetzt sind. Bald scharen sie sich dichter, bald treten sie auseinander, je nachdem, ob ein Gebiet verhältnismäßig zu sehr verkleinert oder vergrößert erscheint.

Zunächst wurden zehn Karten geprüft, die Europa und insbesondere das Mittelmeer betreffen.

\section{Europa von Lopo Homem, um 1550 (Band I, Tafel 25)}

Die Meridian- und Breitenabstände zeigen über dem Mittelmeer und Schwarzen Meer ein erstaunlich regelmäßiges Netz, jedoch mit allgemeiner Aufwölbung gegen Osten. Spanien, Italien, Nordafrika sind gut gezeichnet. Dagegen erscheint Nordeuropa ungenau; insbesondere sind Nord- und Ostsee stark verkürzt.

\section{Europa von Sebastian Lopes, um 1555 (Band IV, Tafel 391)}

Die Breitenkreise zeigen die allgemeine Neigung, im Osten mittels einer Aufbiegung anzusteigen. Die Meridiane werden gegen Osten schräger, indem sie von oben links nach unten rechts streichen. Siehe Abb.1.

3. Mittelmeer von Diogo Homem, 1558 (Band II, Tafel 102)

Auch hier ergibt sich, daß die Karte nicht genau nach Norden orientiert ist, sondern um einige Grad nach Nordosten. 


\section{Mittelmeer von Diogo Homem, 1559 (Band II, Tafel 111)}

Das Gradnetz ist wiederum im Gegenuhrzeigersinn gedreht. Die Küsten von Italien und Nordafrika liegen sich passend gegenüber; dagegen sind die West- und Ostküste Italiens kulissenartig gegeneinander verschoben. Abstände der Inseln vom Festland sind nicht genau getroffen.

5. Schwarzes Meer von Diogo Homem, 1559 (Band II, Tafel 113)

Die Buchten lassen sich gut verfolgen. Die Achse des Meeres ist gedreht; das Ostende liegt zu weit nördlich.

6. und 7. Adriatisches und Ägäisches Meer von Diogo Homem, 1559 (Band II, Tafeln 114 und 115)

Es lassen sich genaue Linien ziehen. Beide Meere sind nordöstlich orientiert.

8. Europa von Diogo Homem, 1561 (Band II, Tafel 119)

Wieder zeigt das Mittelmeer die nordöstliche Orientierung. Beim Schwarzen Meer nimmt sie zu.

9. Östliches Mittelmeer und Schwarzes Meer von Diogo Homem, 1574 (Band II, Tafel 153)

Die Breitenkreise steigen ostwärts an. Die Meridiane stehen senkrecht zu ihnen.

10. Europakarte von Ferdinand Vaz Dourado, 1580 (Band III, Tafel 319)

Nord- und Ostsee liegen gegenüber dem Mittelmeer zu weit westlich und haben eine zu kleine Ostwest-Ausdehnung. -

Im ganzen ergibt sich, daß gerade auch die bestbekannten Gebiete nicht in Nordorientierung wiedergegeben sind, sondern daß die Kartenachse immer im Gegenuhrzeigersinn gedreht ist.

Weiter wurden acht Karten des Atlantischen Ozeans untersucht.

\section{Karte von Pedro Reinel, 1535 (Band I, Tafel 14)}

Links Amerika, rechts Europa und Afrika. Die Breitenkreise laufen waagrecht über den Atlantik und biegen sich nur im Mittelmeer nach Osten zu aufwärts. 


\section{Karte von Sebastian Lopes, 1570 (Band IV, Tafel 407)}

In der Mitte Amerika, rechts Europa und Afrika. Auch hier liegen Äquator und Wendekreise auffallend richtig. Die Meridiane verlaufen in Amerika von oben rechts nach unten links, in Europa und Afrika von oben links nach unten rechts.

\section{Südlicher Atlantik von Antonio Sanches, 1641 (Band V, Tafel 530)}

Die Breitenkreise verlaufen gleichmäßig, sinken jedoch im südlicheren Südamerika ab.

\section{Atlantik von Johann Teixeira Albernaz I, 1646 (Band IV, Tafel 508)}

Afrika ist nordöstlich, Amerika nordwestlich orientiert. Die Ostküste Nordamerikas ist viel zu schräg gerichtet.

\section{Atlantik von Johann Teixeira Albernaz II, 1675 (Band V, Tafel 549)}

Die Breitenkreise stimmen recht gut. Die Meridiane streben nach Norden gegeneinander und scharen sich im Nordatlantik enger zusammen als zwischen Südamerika und Afrika. Die Atlantikküsten Nord- und Südamerikas schließen, von Mexiko aus betrachtet, auf der heutigen Karte einen Winkel von $60^{\circ}$ ein, auf der alten Karte einen Winkel von $45^{\circ}$. Die Ostküste Nordamerikas tritt zuwenig nach Westen, die Nordküste Südamerikas zuwenig nach Süden zurück. Diese Küsten sehen aus wie eine zu stark zugeklappte Schere; Nord- und Südamerika sind einander zugedreht. Die Meridiane, die im Meer vor Nordamerika schräg von oben rechts nach unten links ziehen, erreichen vor Mittelamerika ihren westlichsten Punkt und biegen weiter südlich wieder nach Osten zurück. Siehe Abb. 3 und 4.

\section{Mittelamerika von Sebastian Lopes, um 1565 (Band IV, Tafel 406)}

Das Netz ist in keiner Richtung einheitlich verdreht. Äquator und Wendekreise stimmen. Die Küsten sind genau umrissen. Örtliche Verzerrungen beruhen auf ungenauer Entfernung der Inseln vom Festland.

7. Mittelamerika von Ferdinand Vaz Dourado, 1568 (Band III, Tafel 251)

Wohl erleiden die Längen- und Breitenkreise im einzelnen starke Biegungen; die Nordorientierung ist jedoch gut getroffen. 
8. Mittelamerika von Ferdinand Vaz Dourado, 1570 (Band III, Tafel 261)

Die Küsten von Atlantik und Pazifik sind gegeneinander verschoben. Sonst aber ist die Karte gut und unverdreht. -

Zusammenfassend läßt sich sagen, daß auf den portugiesischen Seekarten die Atlantikküste Nordamerikas eine Drehung im Uhrzeigersinn, jene Südamerikas eine Drehung im Gegenuhrzeigersinn aufweist, während Mittelamerika genau nach Norden orientiert ist.

Es wurde auch die Atlantikkarte des türkischen Admirals Piri Re'is herangezogen, die auf eine Küstenaufnahme des Kolumbus selbst zurückgehen soll. Wenn man den Äquator gerade einzieht, so scheinen die nördlichen Breiten von 10, 20, 30 Grad gegen Amerika immer steiler nach Norden hinaufzustreben. Wenn wir uns die Meridiane senkrecht dazu denken, so bestätigt sich die Drehung im Uhrzeigersinn.

Schließlich wurden drei Karten des Ostens und drei Weltkarten mit Verzerrungsgittern versehen.

\section{Ostasien von Diogo Homem, 1558 (Band II, Tafel 105)}

Die Umrisse von Indien, Hinterindien, Malakka, Sumatra, China und Korea sind gut gezeichnet, ebenso die Nordküste Javas und die Westküste Borneos. Die Linien brechen ab, wo das Wissen aufhört. Die Meridiane und Breitenkreise zeigen einen geordneten Verlauf. Bei Malakka und Sumatra ist die Orientierung nordwestlich.

2. Arabien und der Indische Ozean von Ferdinand Vaz Dourado, 1575 (Band III, Tafel 305)

Über Arabien steigen die Breitenkreise noch ostwärts an; die Meridiane stehen senkrecht dazu. Im Indischen Ozean aber ziehen die Meridiane von oben rechts nach unten links.

3. Ostafrika bis Indien von Johann Teixeira Albernaz I, 1643 (Band IV, Tafel 503)

Auch hier verliert sich die Drehung im Gegenuhrzeigersinn bei Arabien.

4. Weltkarte eines Unbekannten (von Mittelamerika bis Ostasien reichend), 1502 (Band I, Tafel 4)

In weiter Schüsselform schwingen die Breitenkreise über Atlantik, Europa und Nordafrika; der Schüsselgrund ist südlich von Spanien. In 


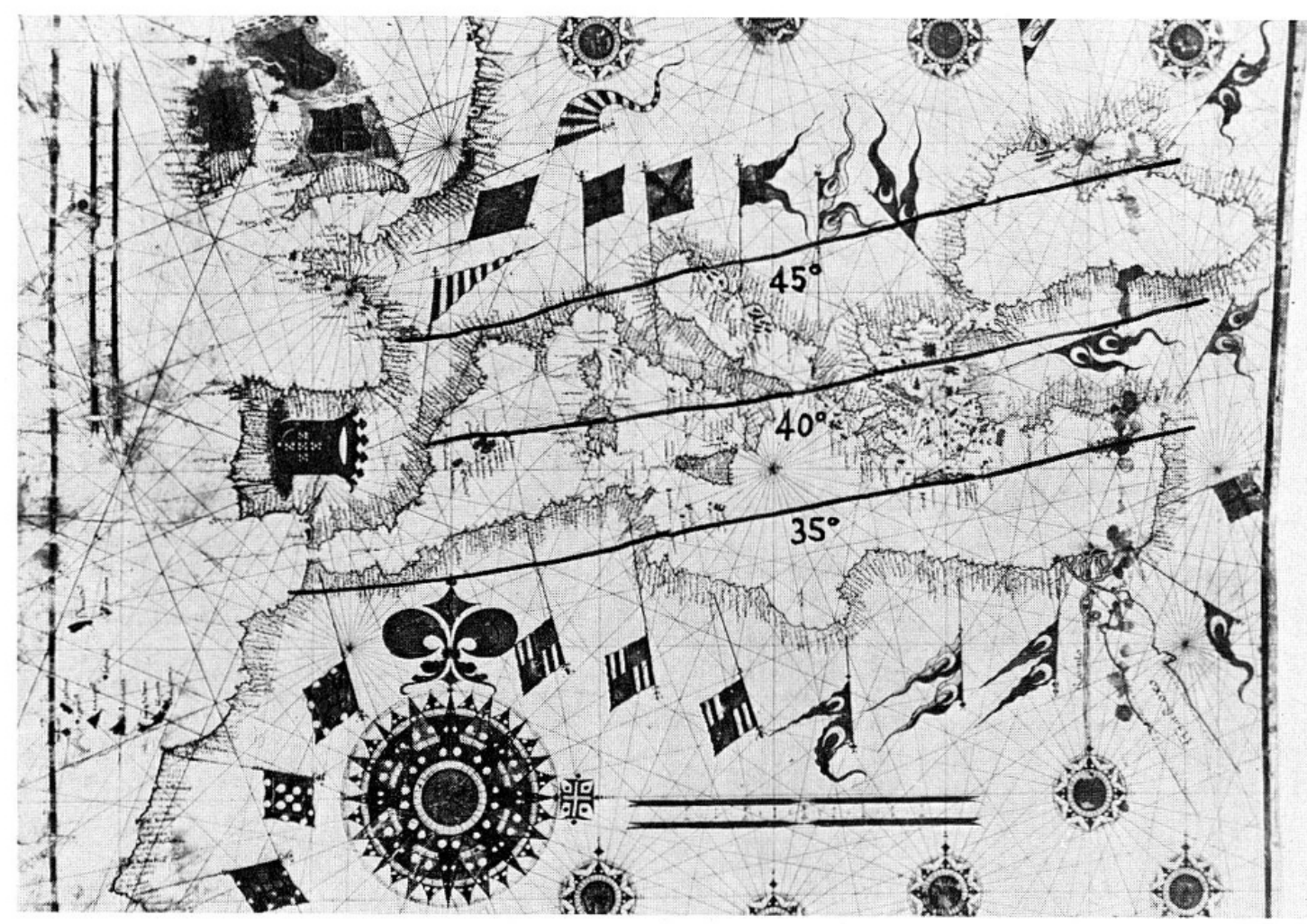

Abb. 1. S. Lopes, um 1555 (Tafel 391). Drehung der Mittelmeerachse im Gegenuhrzeigersinn

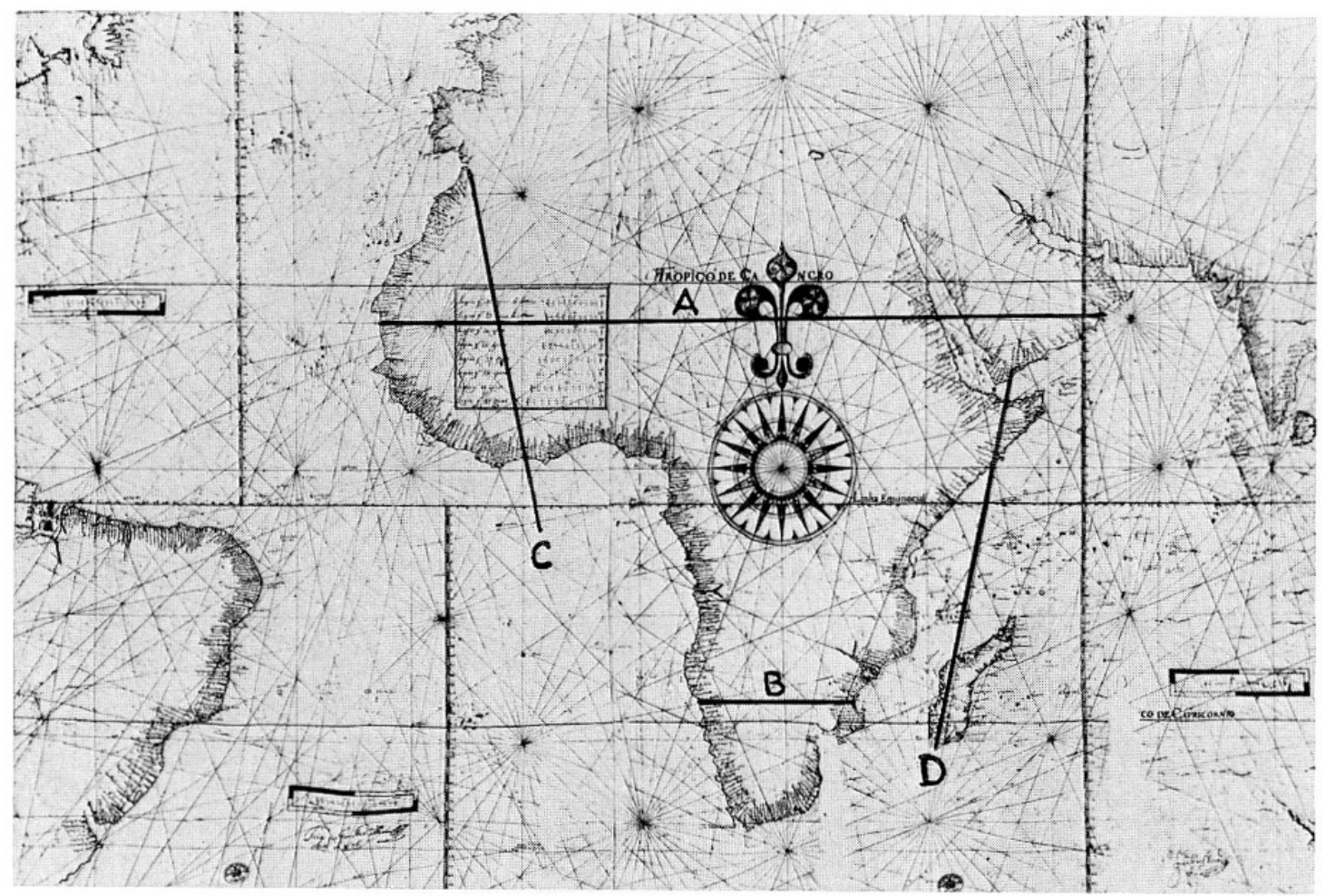

Abb. 2. Joh. Teixeira Albernaz II, 1676 (Tafel 550). $\mathrm{A}=20^{\circ} \mathrm{N}$, zu lang; $\mathrm{B}=20^{\circ} \mathrm{S}$, zu kurz; $\mathrm{A}: \mathrm{B}=4,74$ statt 3,38. C und D = Verlauf der Meridiane, Scharung bei Südafrika. Die atlantische Küste ist im Gegenuhrzeigersinn, die indische im Uhrzeigersinn gedreht 
Im 17. Jahrhundert erscheint die Nordamerikaküste im Uhrzeigersinn und die Südamerikaküste im Gegenuhrzeigersinn gedreht

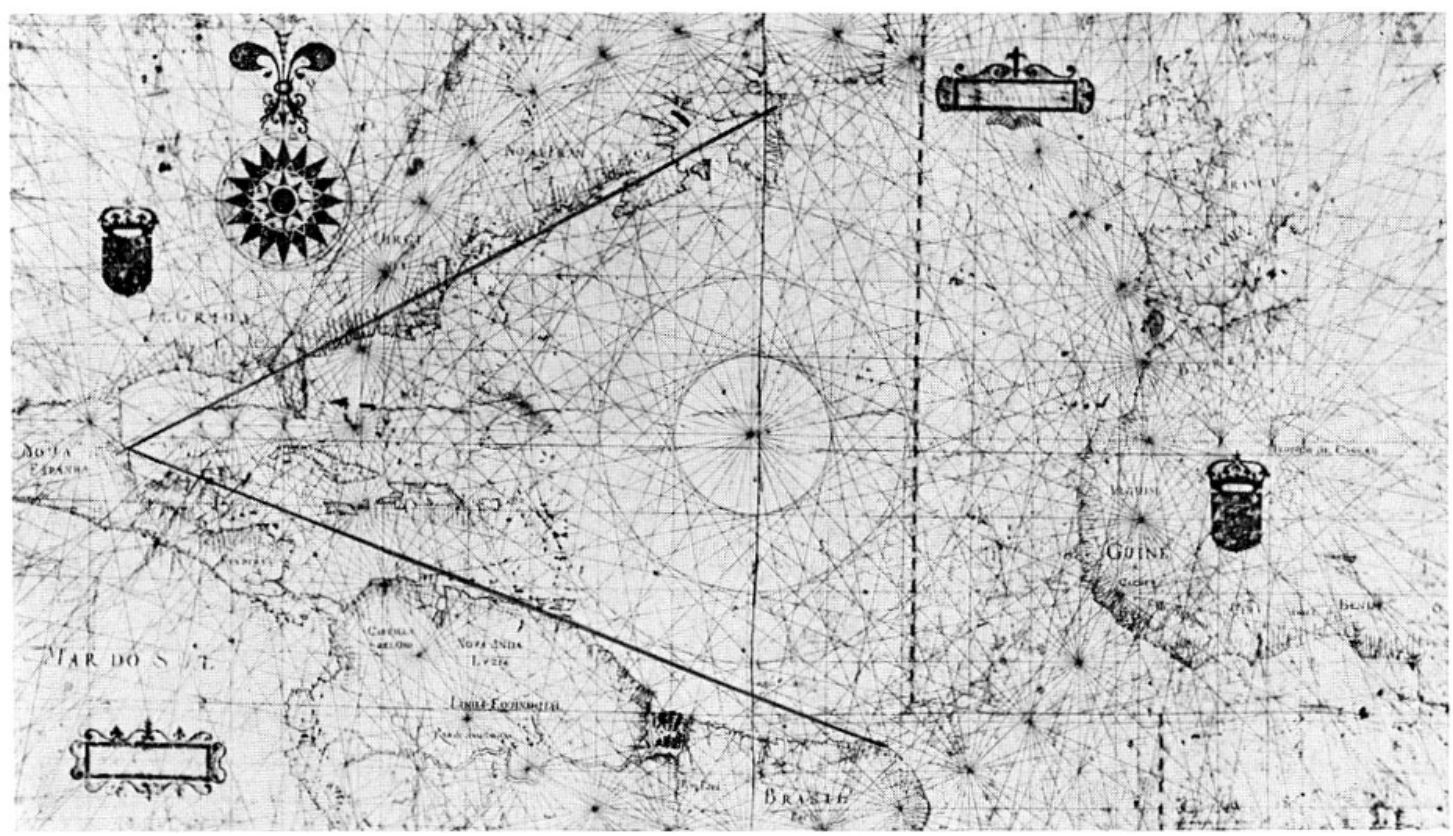

Abb. 3. Johann Teixeira Albernaz II, 1675 (Tafel 549)

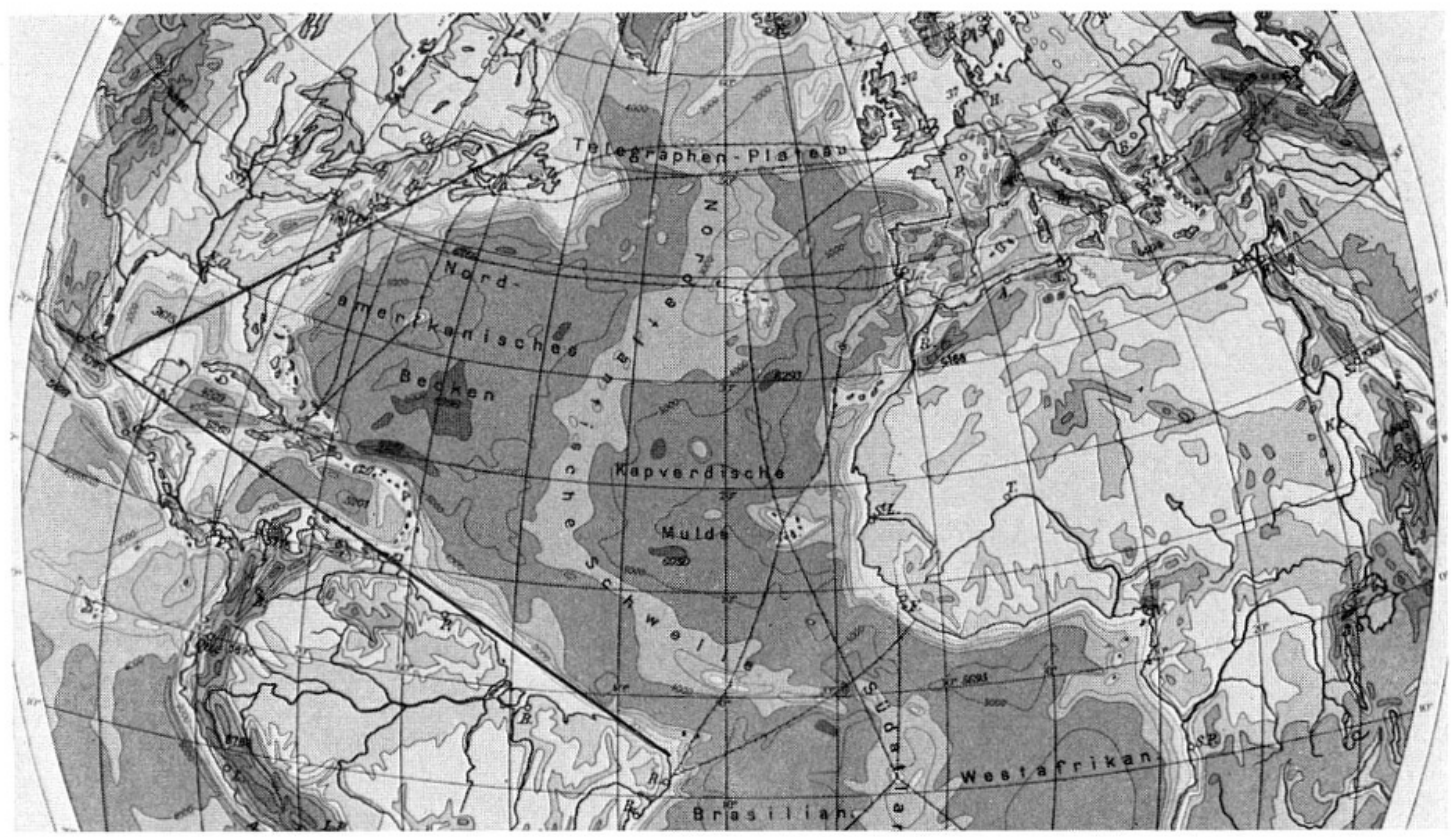

Abb. 4. Eduard Imhof, 1962 (Schweizerischer Mittelschulatlas, S. 108) 
Europa und Afrika ziehen die Meridiane von oben links nach unten rechts, in Indien und im Indischen Ozean umgekehrt von oben rechts nach unten links. Bei der Südspitze Afrikas scharen sie sich von beiden Seiten zusammen.

5. Weltkarte von Lopo Homem, 1554 (Band I, Tafel 27)

Äquator und Wendekreise stimmen. Das Mittelmeer ist nordöstlich, Ostasien nordwestlich orientiert.

\section{Karte von Johann Teixeira Albernaz II, 1676 (Band V, Tafel 550)}

Die Karte reicht von Brasilien über Afrika bis Vorderindien. Die Breitenkreise verlaufen ziemlich waagrecht. Genau stimmen Äquator und Wendekreise. Die Meridiane treten bei Südafrika näher zusammen und streben nach Norden leicht auseinander wie ein Blumenstrauß. Südafrika ist zu schmal, Nordafrika zu breit dargestellt. Siehe Abb. 2. -

Während Arabien und die Westküste Afrikas noch im Gegenuhrzeigersinn gedreht sind, ergibt sich somit für die Gebiete weiter im Osten eine Drehung im Uhrzeigersinn.

Nach der Betrachtung aller dieser Karten stellen wir fest, daß die Küstenorte unter dem Äquator und an den Wendekreisen stimmen. Dort muß die Breitenbestimmung genau gewesen sein. Vielbefahrene Küsten stehen sich richtig gegenüber, so Sizilien und Nordafrika. Dagegen sind zwei Küsten beidseits einer Landmasse manchmal kulissenartig verschoben, so in Süditalien, Mittelamerika und Südafrika. Inseln sind oft in der Form getroffen, aber in der Lage verschoben, Sardinien zu östlich, Malta zu westlich, Madagaskar zu südlich gezeichnet.

Früh war die Höhe der Darstellung erreicht; die späteren Seekarten waren nicht besser als die früheren. Anfangs gab es nur wenige Kartenzeichner; aber sie waren Meister wie Lopo und Diogo Homem. Sie stützten sich auf Originalquellen, auf Logbücher und Skizzen der Entdecker. Später wurde die Kartenherstellung leichter; mancher zeichnete die Vorlagen nach. Dabei wurde das Bild nicht besser, sondern ungenauer. Der unwirkliche Südkontinent, von Abraham Ortelius und Gerhard Mercator angenommen, erscheint auf portugiesischen Seekarten erst nach 1590.

Das Merkwürdigste aber bleiben die Orientierungsfehler. Nordamerika, der Indische Ozean und Ostasien sind im Uhrzeigersinn gedreht, dagegen Europa, das Mittelmeer, das Schwarze Meer, Nord- und Westafrika sowie 
Südamerika im Gegenuhrzeigersinn. Mittelamerika und das ferne Ostasien sind nicht verdreht. Wie kommt es, daß gerade die bekanntesten Küsten falsch orientiert sind, während jene fernen Gestade sich richtig ins Gradnetz fügen?

\section{Deutung der Verzerrungen}

Eine Betrachtung, wie die Seekarten entstanden und mit welchem Hilfsmittel sie aufgenommen worden sind, kann uns vielleicht Aufschlu $\beta$ geben.

Die Kreuzzüge hatten den Kompaß nach Europa gebracht. In besondern Büchlein, den Portulani, wurden Kompaßrichtung und Entfernungsschätzung von Küstenpunkt zu Küstenpunkt vermerkt. Nach diesen Angaben entstanden jene Seekarten.

Um 1300 wurde in Amalfi bei Neapel die Kompaßform erfunden, die sich für die Seefahrt als besonders geeignet erwies. Ein rundes Papierblatt mit aufgezeichneter Windrose trug in der Mitte ein Metallhütchen und schwebte auf einem Stift. Die Magnetnadel war in Form einer Drahtschlaufe unten am Windrosenblatt befestigt. Das Auge erblickte von oben die Scheibe mit dem bunten Stern der Windstriche und am Ende des nördlichen Windstrichs das Bild einer Lilie, die ruhig nach Norden einspielte. Mit solchen Kompassen, deren Gehäuse allmählich eine cardanische Aufhängung erhielt, waren die Seefahrer im 16. und 17. Jahrhundert ausgerüstet. Pedro de Medina beschreibt diesen Kompaß in der Art de naviguer 1561, Georges Fournier in seiner Hydrographie 1643.

Die Seekarten tragen kein Längen- und Breitennetz, dafür aber auf den Meeresflächen ein Gewebe von Windstrichen. Diese strahlen von gemalten Windrosen aus, die in regelmäßigem Kranz über das Kartenblatt verteilt sind. Die Haupt- und Nebenwindrichtungen werden durch Farben unterschieden, die sich bei der Windrose des Kompasses wiederfinden. Dadurch wurde das Segeln nach der Karte erleichtert. Der Kompaß ermöglichte die Entstehung der Karte, und diese wiederum leitete das Segeln mit dem Kompaß, indem man nach ihm die Karte richtete und den Windstrich, längs dem man fahren mußte, daraus ablas.

Vom ersten Augenblick an, wo man den Kompaß zur Seefahrt heranzog und Portulani anlegte, machte sich eine zunächst verborgene, dann früh erkannte Eigenschaft des Magneten geltend, die man nicht auszuschalten vermochte. Die Nadel wies nicht genau nordwärts, sondern zeigte eine von Ort zu Ort wechselnde Abweichung oder Deklination. Aus der Tatsache 
der Abweichung ergab sich die Verdrehung, aus der örtlichen Verschiedenheit der Abweichung die Verbiegung der Karten.

Der erste, der sich dies klar überlegte, war Gerhard Mercator. Er spricht sich in einem Brief an Antoine Perrenot 1546 darüber aus. Angenommen, wir segelten bei östlicher Deklination von Gibraltar aus nach dem östlichen Windstrich, so werden wir nicht die wahre Ostrichtung einschlagen, sondern von ihr nach Süden abweichen. Der Punkt der afrikanischen Küste, auf den wir stoßen, wird uns genau östlich von Gibraltar zu liegen scheinen; wir werden ihn auf der Karte auf dieselbe Breite zeichnen, obschon er südlicher liegt. Wir dürfen es nicht ändern; sonst kann der nächste nicht nach unserer Karte segeln. Die Gebiete des Ostens werden auf der Zeichnung gegenüber dem Ausgangspunkt nach Norden emporgehoben, und zwar um so mehr, je größer die östliche Abweichung wird.

Denken wir uns dagegen von England westwärts gegen Nordamerika fahrend (auch dieses Beispiel stammt von Mercator), so geraten wir über eine Linie ohne Abweichung hinaus in ein Gebiet westlicher Mißweisung. Wir segeln also bei Einhaltung des Westkurses nicht mehr westlich, sondern steuern unvermerkt weiter nach Süden, und dort, wo wir landen, ergibt die Bestimmung der Polhöhe einen kleineren Wert als zu Hause. Dennoch müssen wir den erreichten Punkt auf derselben Breite wie den Ausgangshafen eintragen, denn falls ein nächster an jenen Punki der Küste gelangen will, so muß er genau dem Weststrich der Windrose folgen. Auf einigen Karten wurde daher ein Breitenmaßstab für Europa und ein zweiter, nach Norden hinaufgerückter, für Nordamerika eingezeichnet. Schon Mercator erwähnt dies, und William Borough führt es 1581 in seinem Discourse of the Variation näher aus. Wenn man von der Südwestspitze Englands, von den Scilly-Inseln auf $50^{\circ}$ Breite, ausgehe, erreiche man bei genauer Westfahrt das Kap Race an der Südspitze Neufundlands auf $46^{\circ}$ Breite und brauche daher eine doppelte Breitenskala (a double skale of latitude). Da bei den englischen Kompassen die magnetisierte Drahtschlaufe nicht genau unter dem Nordpunkt der Windrose angebracht war, sondern regelmäßig zwischen 5 und $6^{\circ}$ nach Osten versetzt wurde, weil man so dem Fehler abhelfen wollte, den die damals in Europa herrschende Ostabweichung bewirkte, so fuhr man im Gebiet der Westabweichung um so falscher. Wenn man die Drähte nicht abdrehte, so langte man drüben auf $49^{\circ}$ Breite an, wie Borough schreibt.

Robert Norman berichtet 1581 in The newe Attractive, daß die Mittelmeerkompasse die Drähte unversetzt trugen. Die Spanier und Portugiesen, 
Franzosen und Engländer dagegen versetzten sie für andere Fahrten um einen halben Windstrich, also um $5^{5} / 8^{\circ}$. Borough erhebt den Vorwurf, die Portugiesen hätten die Karten der Meeresbecken einfach zusammengesetzt. Aber im Mittelmeer könne man danach nur mit Mittelmeerkompassen, im Atlantik nur mit versetzten Kompassen segeln. Daß man im Mittelmeer keine Korrektur an den Kompassen anbrachte, lag daran, daß die Küsten bereits aufgenommen waren, bevor man mit der Ostabweichung vertraut war. Darum ist die Abdrehung der Mittelmeerachse auf den Karten auch besonders stark.

Dem Umstand, daß die Abweichung sich von Ort zu Ort änderte, wußte man nicht zu begegnen. Wenn man die Drähte gegenüber der Windrose laufend versetzte, stiftete dies nur Verwirrung. Die Verbindung mußte starr erhalten bleiben, und die Karte mußte verdreht sein, wenn sie der Schiffahrt dienen sollte. Selbst die Erkenntnis des 17. Jahrhunderts, daß die magnetische Abweichung nicht nur von Ort zu Ort, sondern auch im Lauf der Zeiten schwanke, gab den Kompaßkarten nicht sogleich den Todesstoß. Allmählich aber verloren sie wegen dieser Erscheinung ihren praktischen Sinn und erhielten dafür ihren geschichtlichen Wert. Denn es läßt sich (wie Oscar Peschel in seiner Geschichte der Erdkunde dargelegt hat) heute aus der Orientierung dieser alten Seekarten wiederum zurückschließen auf die Deklinationswinkel, die zur Zeit der ihnen zugrunde liegenden Portulani geherrscht haben. Da die späteren Karten auf die früheren zurückgehen, so werden wir dadurch in das 16. oder selbst in das 15. Jahrhundert verwiesen. Die Versetzung der Drähte von 5 bis $6^{\circ}$ bewirkte nur eine geringe Änderung; wir lassen sie hier beiseite.

Überlieferte Messungen bestätigen uns, daß zwischen 1520 und 1540 in Wien $4^{\circ}$, in Landshut $8^{\circ}$, in Ingolstadt $10^{\circ}$, in Nürnberg $10^{1 / 4^{\circ}}$, in Danzig $13^{\circ}$ östliche Abweichung herrschte. Ein wertvolles Verzeichnis von $43 \mathrm{Ab}-$ weichungswerten rings um die Erde sammelte der Geograph Petrus Plancius. Es wurde von Simon Stevin 1599 in De Havenvinding veröffentlicht.

Aus allen Angaben schließt man, daß im 16. Jahrhundert eine Linie ohne Abweichung von Mittelamerika her mitten durch den Atlantischen Ozean zum Nordkap aufstieg, in mächtiger Schlinge ganz Europa und ein Stück Asien umfaßte, südwärts durch Afrika lief und es am Südende beim Nadelkap verließ. Im Innern dieser Schlinge herrschte östliche Abweichung, also in Südamerika, in Europa, im Mittelmeer, im Schwarzen Meer, in Afrika. Außerhalb der Schlinge aber wurde die Abweichung westlich, so im Meer vor Nordamerika, im Indischen Ozean und in Ostasien. Erst im fernen 


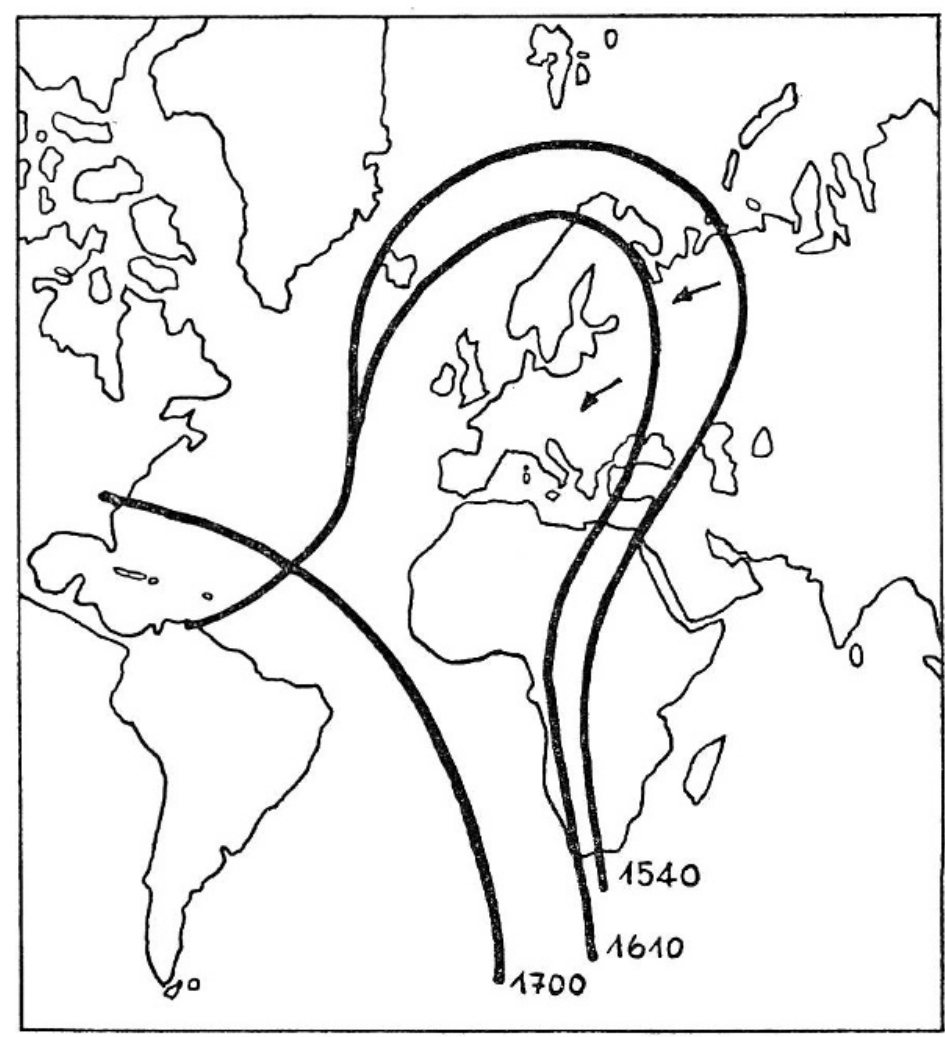

Die Linie ohne magnetische Abweichung verlief quer durch Mittelamerika und längs durch Afrika. Sie hat sich nach Westen verschoben, die Schlinge über Europa sich zugezogen. Für 1540 und 1610 zeichnete die NullIsogone nach alten Meßwerten Willem van Bemmelen (De Isogonen in de XVIde en XVIIde Eeuw, Utrecht 1893). Ich vermute, daß auch Arabien von ihr umfaßt wurde. Für 1700 ist sie von Edmond Halley bestimmt worden (Gustav Hellmanns Neudrucke, Nr.4)

Ostasien und bei Australien traf man wieder auf eine Linie ohne Abweichung. - Dies aber gibt uns den Schlüssel in die Hand, um die rätselhaften Verdrehungen der portugiesischen Seekarten zu deuten.

Als allgemeine Regel läßt sich erkennen, daß bei östlicher magnetischer Abweichung eine Drehung der Küsten und Meeresachsen im Gegenuhrzeigersinn erfolgt ist, während westliche Abweichung eine Drehung im Uhrzeigersinn hervorrief. Mittelamerika und das ferne Asien, wo die Abweichung gegen Null strebte, sind nicht verdreht. Nord- und Südamerika aber mußten um die waagrechte Mittelachse (in der zufällig die Linie ohne Abweichung verlief) einander zugedreht werden wie eine nach rechts zuklappende Schere. West- und Ostafrika mußten um das Nadelkap voneinander abgedreht werden wie eine nach oben auseinanderklappende Schere. Daher scharen sich die Meridiane um Südafrika dichter. Auch im Nordatlantik scharen sie sich dichter, weil Nordamerika und Nordeuropa einander zugedreht sind. So ordnet sich die anfangs verwirrende Fülle der Verzerrungserscheinungen unter das einfache Gesetz: Ostabweichung des Magneten bewirkt auf der Karte eine Drehung im Gegenuhrzeigersinn, Westabweichung eine Drehung im Uhrzeigersinn. 
Unterlagen zum 1. Teil

Portugaliae Monumenta Cartographica, 5 Bände, Lissabon 1960.

Yusuf Akçura, Piri Re’is, Istanbul 1935.

Unterlagen zum 2. Teil

Pedro de Medina, L'art de naviguer, Lyon 1561 (6. Buch).

Georges Fournier, Hydrographie, Paris 1643 (11.Buch).

Gustav Hellmann, Neudrucke von Schriften und Karten über Meteorologie und Erdmagnetismus, Nr.10, Berlin 1898. Darin: Brief von Gerhard Mercator (Löwen, 23. Februar 1546); Robert Norman, The newe Attractive, London 1581 (10. Kapitel); William Borough, A Discourse of the Variation of the Compasse, or Magneticall Needle, London 1581 (11. Kapitel); Simon Stevin, De Havenvinding, Leiden 1599 (Tabelle von Plancius).

Oscar Peschel, Geschichte der Erdkunde, 2. Auflage, München 1877, S. 216 f.

Atlas zu Alexander von Humboldts Kosmos, Stuttgart 1860 (Tafel 13 zeigt den Verlauf der Linien ohne magnetische Abweichung für 1600). Die dortige Zeichnung beruht auf dem Atlas zu: Christopher Hansteen, Untersuchungen über den Magnetismus der Erde, Christiania 1819. Auf Grund weiterer Meßwerte wurden die Linien von Willem vaN BemMelen genauer verfolgt (De Isogonen in de XVIde en XVIIde Eeuw, Utrecht 1893), der sie für 1540, 1580, 1610,1640, 1665 und 1680 zeichnete. Danach hatte die NullIsogone, obschon sie westwärts gewandert war, um 1610 die Westküste Afrikas noch nicht erreicht, was mit unserem Befund übereinstimmt. 